\title{
ORIENTAÇÃO DE ENFERMAGEM PARA A ALTA HOSPITALAR DO PACIENTE NEOPLÁSICO*
}

Ieda Maria Leal da Cruz', Maria de Fátima Mantovani²

'Enfermeira. Mestre em Enfermagem. Hospital de Clínicas da Universidade Federal do Paraná. Curitiba-PR-Brasil. ${ }^{2}$ Enfermeira. Doutora em Enfermagem. Universidade Federal do Paraná.Curitiba-PR-Brasil.

RESUMO: Estudo do tipo metodológico que objetivou elaborar orientações sistematizadas de enfermagem para alta hospitalar do paciente neoplásico. O modelo proposto para as orientações fundamentou-se na Teoria das Necessidades Humanas Básicas de Horta. Participaram 20 pacientes adultos com diagnóstico de câncer, em pronto atendimento de hospital de ensino em Curitiba, Paraná, no período de março a maio de 2013. Os dados foram coletados mediante entrevista semiestruturada e, a partir de análise de conteúdo, emergiram as categorias psicobiológicas, psicossociais e psicoespirituais, as quais subsidiaram a construção de 10 orientações sistematizadas. Conclui-se que materiais educativos possibilitam ao enfermeiro, dentre outros benefícios, identificar as reais necessidades dos pacientes, avaliar a compreensão de orientações de cuidados e, consequentemente, contribuir para a continuidade do tratamento em domicílio.

DESCRITORES: Neoplasias; Cuidados de enfermagem; Alta do paciente.

\section{NURSING ADVICE FOR THE DISCHARGE FROM HOSPITAL OF THE NEOPLASTIC PATIENT}

\begin{abstract}
This study, of the methodological type, aimed to elaborate systematized nursing advice for the discharge from hospital of the neoplastic patient. The model proposed for the guidance is based on Wanda Horta's Basic Human Needs Theory. The participants were 20 adult patients diagnosed with cancer, receiving treatment in an emergency care center at the teaching hospital in Curitiba, Paraná, in the period March - May 2013. The data were collected through semistructured interviews and, based on content analysis, the psychobiological, psychosocial and psychospiritual categories emerged, which formed the basis for the construction of 10 systematized items of advice. It is concluded that educational materials allow the nurse - among other benefits - to identify the patients' real needs, to evaluate the understanding of care advice, and consequently to contribute to the continuity of the treatment in the home.
\end{abstract}

DESCRIPTORS: Neoplasias; Nursing care; Patient discharge.

\section{ORIENTACIÓN DE ENFERMERÍA PARA EL ALTA HOSPITALAR DEL PACIENTE NEOPLÁSICO}

RESUMEN: Estudio del tipo metodológico cuyo objetivo fue elaborar orientaciones sistematizadas de enfermería para alta hospitalar del paciente neoplásico. El modelo propuesto para las orientaciones se ha fundamentado en la Teoría de las Necesidades Humanas Básicas de Horta. Participaron 20 pacientes adultos con diagnóstico de cáncer, en atención de emergencia de hospital de enseñanza en Curitiba, Paraná, en el periodo de marzo a mayo de 2013. Los datos fueron obtenidos por medio de entrevista semiestructurada y, del análisis de contenido, resultaron las categorías psicobiológicas, psicosociales y psicoespirituales, las cuales subsidiaron la construcción de 10 orientaciones sistematizadas. Se concluye que materiales educativos posibilitan al enfermero, entre otros beneficios, identificar las reales necesidades de los pacientes, evaluar la comprensión de orientaciones de cuidados y, consecuentemente, contribuir para la continuidad del tratamiento en domicilio.

DESCRIPTORES: Neoplasias; Cuidados de enfermería; Alta del paciente. 


\section{INTRODUÇÃO}

A Organização Mundial da Saúde (OMS) esclarece que os países em desenvolvimento, entre eles o Brasil, serão os mais afetados por casos novos de câncer, projeta para o mundo 27 milhões os casos novos da doença para 2030 e 17 milhões de mortes. O Instituto Nacional do Câncer (INCA) publicou, em novembro de 2013, a estimativa de 580 mil casos novos da doença para 2014. Há previsão de 69 mil casos novos de câncer de próstata; 57 mil de mama e 27 mil de pulmão. Para o Ministério da Saúde e para o INCA estes números resultam de vários fatores, dentre eles, o envelhecimento da população. Destacam que conhecer estas estimativas reforçam a necessidade de planejamento e gestão para prevenção, detecção e oferta de tratamento adequado $^{(1)}$.

Dentre as formas de tratamento oncológico o cirúrgico pode se revelar muito assustador e temido. Embora o paciente receba informações e orientações há relatos de não terem compreendido o que lhes foi falado ou mostrado, de tal forma que ao longo da internação e no preparo para a alta há muito a ser esclarecido pelo enfermeiro. Deste modo, há demanda de esclarecimentos acerca de procedimentos diagnósticos e terapêuticos ${ }^{(2)}$.

Em outro aspecto, pesquisa de revisão integrativa constatou que há tendência à diminuição do tempo de internação de pacientes cirúrgicos, fato que pode interferir negativamente nas atividades educativas que os preparem para a alta hospitalar. A literatura consultada conclui que a enfermagem deve prover informações, sendo este o foco principal para minimizar ansiedade, combinando informes orais e escritos como estratégia para a realização de cuidados em domicílio $^{(3)}$.

Deste modo, por ocasião da alta hospitalar o planejamento para a continuidade do tratamento será benéfico para o paciente e seus familiares, mas para isso é importante a troca de informações efetivas entre este e a equipe de enfermagem, para o seguimento correto do cuidado por parte do paciente $^{(4)}$.

Contudo, a realidade tem mostrado que o processo de produção de material educativo raramente é descrito e o resultado final é pouco avaliado. É importante esclarecer suas finalidades: auxiliar pacientes e familiares sobre a recuperação e estimular o autocuidado; uniformizar informações e orientações; auxiliá-los a entender sua trajetória de adoecimento( ${ }^{(2)}$.

Os materiais educativos, quaisquer que sejam, devem ter orientações com rigor científico ao paciente neoplásico, porém devem ir além de aspectos biológicos, inserindo questões importantes relacionadas às necessidades psicossociais e espirituais, pois de outro modo o material educativo será meramente generalista. Assim, devem contribuir para minimizar as dúvidas referentes ao tratamento farmacológico e não farmacológico e servir de base para estimular a adesão ao tratamento proposto, evitando as reinternações.

Considerando o contexto de gravidade da neoplasia na sociedade e necessidade contínua da participação do enfermeiro no cuidado ao doente oncológico, este artigo tem por objetivo elaborar orientações sistematizadas de enfermagem para alta hospitalar do paciente neoplásico.

\section{METODOLOGIA}

Trata-se de pesquisa metodológica, desenvolvida em um serviço de pronto atendimento adulto, na Unidade de Urgência e Emergência Adulto (UUEA) de hospital público de ensino, na cidade de Curitiba-PR.

A pesquisa metodológica refere-se a investigações dos métodos para obtenção, organização, validação e avaliação de instrumentos e técnicas de pesquisa(5), está associada a caminhos, formas, maneiras e procedimentos para atingir determinado fim ${ }^{(6)}$. O estudo foi realizado em 2 etapas.

Na primeira etapa, utilizou-se a entrevista aos pacientes, tendo como norte as necessidades de cuidados baseado em Horta ${ }^{(7)}$. Segundo Horta as necessidades psicobiológicas são: sono e repouso, nutrição, náusea, eliminações, cuidados corporais, oxigenação, sexualidade, regulação vascular e ambiente. As necessidades psicossociais: segurança, ansiedade, atenção, aceitação, autoestima e autoimagem, autorealização e liberdade e, lazer. As necessidades psicoespirituais: espiritualidade, religiosa ou teológica.

A amostra foi intencional com pacientes adultos, com idade superior a 18 anos e menos 
de 60 anos, com complicações oncológicas, condições de comunicação verbal e disposição para responder as questões de uma entrevista. A saturação da amostra deu-se por repetição de informações.

A coleta de dados ocorreu nos meses de março a maio de 2013 e os depoimentos obtidos foram transcritos e analisados a partir da análise de conteúdo proposta por Bardin ${ }^{(8)}$.

Na segunda etapa da pesquisa metodológica foram elaboradas as orientações aos pacientes em fluxogramas, por considerar que é de fácil entendimento, tanto para a equipe de enfermagem, quanto para o paciente. Para esta construção utilizou-se o programa de informática Office Word 2011®, SmartArt, constituído por elemento gráfico, na forma de diagrama, permitindo a inserção de informações.

O estudo seguiu as normas da Resolução 196/1996 do Conselho Nacional de Saúde e, obteve parecer favorável do Comitê de Ética em Pesquisas do Setor de Ciências da Saúde da Universidade Federal do Paraná, sob o Registro CEP/SD: 1227.152.11.09. O Termo de Consentimento Livre e Esclarecido foi assinado pelos participantes e, em respeito às questões éticas, manteve-se o anonimato dos mesmos durante a transcrição dos dados coletados.

\section{RESULTADOS}

Participaram do estudo 20 pacientes com diagnóstico de câncer que estavam em observação na unidade pesquisada. Destes, 65\% eram homens e $35 \%$ mulheres, com idade entre 21 e 60 anos, tendo como média de 48,6 anos. Os tipos de canceres mais prevalentes foram: câncer de mama, aparelho reprodutor feminino, aparelho gastrointestinal e leucemia.

Dentre os relatos dos pacientes a náusea foi a queixa referida como sensação desagradável de "mal estar no estômago" ligada aos efeitos do tratamento quimioterápico, medicações usadas para aliviar a dor e aos odores presentes no ambiente.

Os participantes queixaram-se de dor, mesmo quando medicados. Durante as entrevistas a dor foi caracterizada tanto pelo relato verbal como pela expressão facial dos pacientes.

Referente à nutrição, a metade dos participantes apresentou queixas relacionadas aos horários das refeições e tipo de dieta. Por outro lado, também relataram alterações no hábito alimentar, como a diminuição do apetite, após o diagnóstico do câncer. Entre outras necessidades afetadas, 14 participantes apresentaram constipação intestinal. Em suas falas os pacientes associaram a eliminação prejudicada ao uso das medicações utilizadas no alívio e controle da dor.

Houve queixa sobre o sono e o repouso, com dificuldade para adormecer ou permanecer dormindo. As queixas tratam inclusive sobre o tipo de cama para o repouso e a dificuldade em acomodar-se.

Dois participantes relataram falta de ar e outros dois apresentaram edema em membros inferiores. Com relação ao cuidado corporal, cinco participantes relataram a necessidade de auxílio para a realização da higiene devido à dor. Também uma participante relatou ter a sua sexualidade prejudicada, com limitações físicas, em decorrência da doença.

No que tange às necessidades psicossociais afetadas, a sensação de incapacidade, insegurança e a incerteza quanto à recuperação foi mencionada por todos. Outras questões abordadas foram à perda do controle sobre suas vidas após o adoecimento; autoimagem alterada, citada por uma participante, que relatou sentirse deteriorando, em função da perda de dentes e cabelos e as transformações do corpo. A falta de lazer foi apontada por 12 participantes de ambos os sexos, referindo aborrecimento pela doença e pela condição de vida que ela tem lhes proporcionado.

Quanto às necessidades espirituais os participantes mencionaram suas preferências religiosas e expressaram a necessidade do apoio espiritual no percurso da doença. Observou-se, a partir dos relatos de quatro participantes, que a expressão da fé e da espiritualidade é muito pessoal, porém, todos relataram o apego a estes sentimentos, com a esperança de sobreviver ao câncer.

As queixas relatadas nos depoimentos dos pacientes foram categorizadas a partir de necessidades humanas básicas:

- Psicobiológicas: Dor oncológica, Náusea, Constipação instestinal, Distúrbio de sono e 
repouso; Dispnéia; Falta de apetite e Dificuldade para realizar cuidados corporais.

- Psicossociais: Ansiedade e angústia; Falta de lazer.

- Psicoespirituais: Necessidade de apoio espiritual.

Com esta categorização foram elaboradas, na forma de fluxogramas, 10 orientações à alta hospitalar do paciente neoplásico:

- Orientação 1 - Dor oncológica: descrevemse medidas preventivas e uso de medicações prescritas;

- Orientação 2 - Náusea: apresentados os cuidados para risco de náusea, em duas situações: quando presente e prevenção para que não ocorra;

- Orientação 3 - Constipação instestinal: estimulase o uso de alimentos e líquidos que facilitem o trânsito instestinal; orienta-se sobre medicamentos;

- Orientação 4 - Distúrbio de sono e repouso: há recomendações sobre ambiente, ingesta hídrica e de medicamentos;

- Orientação 5 - Dispneia: há recomendações sobre ambiente; posição e mobilidade;

- Orientação 6 - Falta de apetite: sugerem-se tipos e formas de apresentação de alimentos;

- Orientação 7 - Dificuldade para realizar cuidados corporais: orienta-se o autocuidado quando possível e uso de medicamentos para dor;

- Orientação 8 - Ansiedade e angústia: propõemse atividades lúdicas, apoio psicológico e da família;

- Orientação 9 - Lazer: sugerem-se atividades ao ar livre e interativas;

- Orientação 10- Espiritualidade: orienta-se sobre a importância de crenças e tradições pessoais e familiares.

\section{DISCUSSÃO}

A faixa de idade dos participantes do estudo, em média 48 anos, corresponde a um período de vida produtiva, a doença acarreta grande mudança no aspecto social e é impactante economicamente para a sociedade. O Atlas de Mortalidade do INCA informa que para o sexo masculino como feminino, as taxas de mortalidade por neoplasias malignas, ocorrem com maior incidência a partir dos 30 anos. Deste modo há a necessidade de desenvolver ações de educação permanente em saúde, qualificação de profissionais e mensagens sobre o câncer ${ }^{(9)}$.

Sobre as necessidades básicas afetadas foi referida a dor oncológica, inclusive com o uso de medicação. A dor acomete 60 a $80 \%$ dos pacientes com câncer, sendo que 25 a 30\% na ocasião do diagnóstico e, 70 a 90\% com doença avançada. A dor é fator determinante de sofrimento, relacionado à doença e à expectativa da morte ${ }^{(10)}$.

Considerando que a dor é uma experiência pessoal e subjetiva, só quem a experimenta pode conhecê-la ${ }^{(11)}$. A avaliação da dor não pode ser subestimada, deve ser realizada periodicamente e de forma detalhada quanto a intensidade, duração, características físicas e fatores desencadeantes, para que a terapêutica empregada proporcione o seu alívio completo ${ }^{(12)}$. Deste modo, é possível conhecer as características pessoais de cada paciente facilitando e tornando eficazes as orientações à alta hospitalar.

Pesquisa de revisão sistemática mostra que cuidadores sem apoio levam pacientes do domicílio para serviços de saúde, pois estão incapacitados para o controle da dor. Assim, sugerem intervenções que sejam coerentes com as suas necessidades, envolvendo e capacitando o doente e seus familiares ${ }^{(13)}$.

Referente à nutrição, pacientes relataram alterações na dieta alimentar, após o diagnóstico do câncer. Primeiramente se sugere investigar alterações de paladar, bem como examinar a cavidade oral para identificar possíveis mucosites, estomatites, infecções ou lesões. Todo paciente deve ser avaliado quanto a sua capacidade de se alimentar, grau de desconforto, nível de consciência, preferências alimentares, adaptação a novos alimentos, consistência e horários. Para cuidadores e familiares a falta de apetite pode ser causa de grande sofrimento psicológico, pois a falta ou alteração considerável da vontade de comer indica a gravidade do avanço da doença ${ }^{(14)}$.

As orientações para o cuidador serão evitar odores durante o preparo dos alimentos, uso de condimentos que o doente prefere, redução no tamanho das porções, refeições frequentes que podem melhorar o aporte nutricional. Próteses dentárias bem adaptadas facilitam a alimentação e melhoraram a autoestima do paciente.

Dependendo da fase da doença o paciente sentirá menos sede, recusa a alimentação em função de sintomas como desconforto, dor, obstipação, diarréia, vômitos entre outros. Nesta 
fase convém ouvir o paciente, para combinar métodos diferentes de alimentação, oferecer auxílio, como utensílio mais apropriado, sempre respeitando sua autonomia ${ }^{(14)}$.

Além da fisiopatologia do câncer desencadear alterações metabólicas, as modalidades de tratamento podem provocar outros efeitos: a náusea e constipação instestinal, referidas pelos entrevistados deste estudo como necessidades afetadas. Estes efeitos decorrerem de quimioterapia, radioterapia, medicamentos, desequilíbrios hidroeletrolíticos, mudanças de paladar e odor, obstrução instestinal. Destaca-se que constipação instestinal acomete 60 a $70 \%$ dos pacientes em uso de opiódes, porém seu controle é dificultado pela pouca ingestão de alimentos e líquidos, além do paciente ter náusea e vômito. Deste modo, os pacientes precisam de intervenção nutricional, seja em serviço de saúde como em domicílio, como recursos para mitigar ou prevenir desnutrição ou desidratação(15).

Em pesquisa sobre náusea, vômitos e qualidade de vida de mulheres com câncer de mama em tratamento quimioterápico recomenda-se que, independente das facilidades ou dificuldades para avaliar sinais e sintomas do tratamento quimioterápico, o importante é valorizar as queixas dos pacientes e estabelecer cuidados individualizados. Também, deve-se disponibilizar informações para pacientes e familiares sobre o tratamento e cuidados com efeitos adversos, promovendo assim a melhor qualidade de vida que se possa oferecer, diante das toxicidades gastrointestinais ${ }^{(16)}$.

Outra condição que interfere na vida do doente neoplásico é o sono e o repouso. Os pacientes entrevistados referiram dificuldade para adormecer ou permanecer dormindo. Destacam-se fatores que interferem para suprir estas necessidades básicas: excesso de luz, ruídos contínuos e altos, conversas em excesso, televisão ou rádio ligado, entre outros. Com estas informações e outras referidas pelos pacientes e familiares o enfermeiro poderá realizar orientações pontuais, objetivas, claras e eficientes para promoção do conforto.

Ainda sobre este tema, estudo realizado sobre a qualidade do sono, em pacientes submetidos à cirurgia oncológica, mostra que os distúrbios de sono foram despertar precoce, necessidade de ir ao banheiro, episódios de dor, sensação de calor à noite. Já os episódios de cochilos diurnos estão associados a sono interrompido à noite, contribuindo para a qualidade ruim de sono noturno. Para auxiliar os pacientes os profissionais de enfermagem orientam: manter um horário regular para dormir e acordar; deitar quando estiver realmente com sono; levantar se não conseguir dormir e andar um pouco se possível; não fazer atividade estimulante até sentir sonolência e dormir apenas o necessário; fazer exercícios; fazer um lanche antes de deitar; reduzir o ruído e a luz; evitar nicotina e cafeína; fazer cochilos diurnos breves ${ }^{(17)}$.

A dispnéia foi relatada como necessidade afetada pelos entrevistados nesta pesquisa e sua prevalência é de $55 \%$ nos pacientes com câncer, aumentando com a gravidade da doença ${ }^{(12)}$. Os sintomas de fadiga, cansaço, dispnéia e taquicardia podem ser relacionados à anemia, podendo ser identificada por hemograma, contudo outras investigações podem ser necessárias. Os autores referem que a dispnéia não traz mudanças significativas na qualidade de vida, quando se compara ao início do tratamento e o observado três meses depois, sugerindo uma relação com alguma doença preexistente. Sugerem que atenção individualizada e estendida ao domicilio minimizam efeitos do tratamento oncológico ${ }^{(18)}$.

Foi relatada pelos entrevistados a dificuldade para realizar cuidados corporais, fato que pode estar relacionado ao conjunto de sinais e sintomas acima referidos, como por integridade de pele prejudicada por lesões, pele desnuda, eritema, prurido em decorrência do câncer ou como complicações por baixa mobilidade corporal. A debilidade imposta pela doença diminui as condições de autocuidado, exigindo em domicilio a presença de cuidador que possa reorganizar a rotina doméstica e proporcionar as melhores maneiras de conforto. Neste sentido as recomendações são: mobilidade do paciente, incluindo mudanças de decúbito; higiene corporal com supervisão ou auxílio direto; escovação oral após as refeições; hidratação da pele com óleos e cremes, entre outros. Contudo, as dificuldades financeiras podem estar associadas à condição de cuidado, pois a família passa a ter outros gastos com o paciente, como alimentação e curativos. Neste contexto, as orientações precisam ser relacionadas à realidade vivida pelo doente $\mathrm{e}$ família ${ }^{(19)}$. 
Houve uma necessidade pouco referida: sexualidade prejudicada. Foi relatada por uma participante devido às limitações físicas provocadas pela doença e tratamento. $\mathrm{O}$ adoecimento pelo câncer de mama e os efeitos colaterais advindos do tratamento interferem negativamente na elaboração da imagem corporal e na vida sexual da mulher ${ }^{(20)}$. A sexualidade não é somente a necessidade de consumar o relacionamento sexual, mas também de integrar aspectos emocionais, intelectuais e sociais ${ }^{(21)}$.

A autoimagem pode se configurar como necessidade humana afetada. Pacientes portadores de câncer podem apresentar diferentes reações na maneira como percebem a sua imagem corporal, decorrentes da doença e dos diversos tipos de tratamentos. O conceito de imagem corporal é a figura mental do corpo humano e, o modo pelo qual é apresentado ao outro, sendo referência do homem a si mesmo e ao mundo ${ }^{(22)}$.

O déficit de lazer foi apontado pelos participantes de ambos os sexos como necessidade afetada. Estes referiram aborrecimento relacionado à hospitalização e, muitas vezes à restrição ao quarto. Em estudo sobre avaliação de qualidade de vida, relacionada à saúde de mulheres com câncer de colo uterino, elas mencionaram a televisão como atividade recreativa mais comum. O lazer tem relação direta com fatores individuais, nível de escolaridade e o uso que o paciente fazia do tempo livre antes da doença. Para orientações educativas, que favoreçam o bem estar do paciente, sugere-se atividades de qualquer natureza, pois a falta de lazer pode acentuar a solidão, aparecimento de queixas somáticas e dificuldade de manter relacionamentos interpessoais. A diversão e o entretenimento aliviam tensões, dão uma pausa aos sofrimentos, com efeito benéfico na qualidade de $v^{2} a^{(23)}$.

No âmbito das necessidades psicoespirituais, observou-se a partir dos relatos dos participantes, que a expressão da fé e da espiritualidade é muito pessoal, porém, todos fizeram relação com a esperança de sobreviver ao câncer, que amedronta, mas a fé que renova. Os termos espiritualidade e religião estão relacionados, mas apresentam características diferentes. A espiritualidade é mais ampla e pessoal, relacionada aos valores íntimos, dando sentido à vida, independente da religião ${ }^{(24)}$.

A espiritualidade é uma construção da personalidade de cada indivíduo, que expressa sua identidade e sua história pessoal. Também, a fé em Deus é um sentimento presente na cultura do ser humano, constituindo uma maneira construtiva de pensar. É uma busca contínua de respostas para grandes questões humanas como o significado da existência humana ${ }^{(12)}$.

As referências aqui citadas demostram que os pacientes e familiares, por ocasião da alta hospitalar, esperam do enfermeiro e da equipe de enfermagem informações e orientações confiáveis e objetivas, transmitidas com clareza na linguagem e que se sintam confiantes em proporcionar o melhor cuidado possível no domicílio.

\section{CONSIDERAÇÕES FINAIS}

Cuidar de pacientes portadores de câncer, apesar de causar sofrimento, pode produzir sentimento de gratificação aos enfermeiros. Permanecer ao lado do paciente por alguns minutos, um simples toque ou um gesto de carinho, pode ajudá-lo a valorizar pequenas ações, que podem levá-lo a seguir em frente, a propiciar bem estar, ao tempo de vida que ele tiver.

Muitos fatores foram importantes para a realização deste estudo, sendo que um deles foi utilizar a Teoria das Necessidades Humanas Básicas, de Horta, que permitiu a coleta de dados para a construção de orientações. Outro diferencial foi associar esta mesma teoria à prática do enfermeiro, como suporte frente às intervenções necessárias nas ações do cuidado.

O presente estudo alcançou seu objetivo com a elaboração de orientações para alta hospitalar do paciente neoplásico. Os mesmos podem ser reformulados conforme a demanda de cada serviço e, devem ser vistos como facilitadores, servindo de subsídio ao enfermeiro, para o cuidado ao paciente com câncer, da internação à alta hospitalar.

\section{REFERÊNCIAS}

1. Ministério da Saúde (BR). Instituto Nacional do Câncer - INCA. Estimativas de câncer para 2014. [Internet] [acesso 31 mar 2014.] Disponível: http://www.inca. gov.br/estimativa/2014/

2. Gozzo TO, Lopes RR, Prado MAS, Cruz LAP, Almeida 
AM. Informações para a elaboração de um manual educativo destinado às mulheres com câncer de mama. Esc. Anna Nery. 2012;16(2):306-11.

3. Mata LRF, Napoleão AA. Intervenções de enfermagem para alta de paciente prostatectomizado: revisão integrativa. Acta Paul. Enferm. 2010;23(4):574-79.

4. Souza PMBB. Protocolo de orientações de enfermagem para o paciente com insuficiência cardíaca à alta hospitalar [dissertação]. Rio de Janeiro (RJ): Universidade Federal Fluminense; 2012.

5. Polit DF, Beck CT, Ungler BP. Fundamentos da pesquisa em enfermagem: método, avaliação e utilização. Porto Alegre: ARTMED; 2004.

6. Demo P. Pesquisa e construção do conhecimento: metodologia científica no caminho de Habermas. Rio de Janeiro: Tempo Brasileiro; 1994.

7. Horta WA. Processo de enfermagem. São Paulo: EPU; 1979.

8. Bardin L. Análise de conteúdo. São Paulo: Edições 70; 2011.

9. Ministério da Saúde (BR). Instituto Nacional do Câncer - INCA. Atlas de Mortalidade do Instituto Nacional do Câncer, 2002 [Internet] [acesso 31 mar 2014]. Disponível: http://www.inca.gov.br/atlas/docs/ Atlas_completo.pdf

10. Rangel $\mathrm{O}$, Telles $\mathrm{C}$. Tratamento da dor oncológica em cuidados paliativos. Rev. HUPE. 2012;11(2):32-7.

11. Alves VS, Santos TS, Trezza MCSF, Santos RM, Monteiro FS. Conhecimento de profissionais da enfermagem sobre fatores que agravam e aliviam a dor oncológica. Rev. bras. cancerol. 2011;57(2):199-206.

12. Moraes TM. Como cuidar de um doente em fase avançada de doença. Mundo Saúde. 2009;33(2):23138.

13. Correia I, Frade M. Programa educacional ao cuidador do doente oncológico com dor no domicilio - revisão sistemática da literatura. Repositório Universidade de Évora, 2012 [Internet] [acesso 31 mar 2014]. Disponível: http://hdl.handle.net/10174/6362

14. Academia Nacional de Cuidados Paliativos- ANCP. Manual de cuidados paliativos. Rio de Janeiro: Diagraphic; 2009.

15. Palmieri BN, Moulatet EM, Buschinelli LKO, Pinto-eSilva MEM. Aceitação de preparações e sua associação com os sintomas decorrentes do tratamento de câncer em pacientes de uma clínica especializada. Cad. saude colet. 2013;21(1):2-9.

16. Gozzo TO, Moysés AMB, Silva PR, Almeida AM.
Náuseas, vómitos y calidad de vida en mujeres con cáncer de mama en la quimioterapia. Rev. Gaúcha Enferm. [Internet] 2013;34(3):110-6 [acesso em 04 abr 2014] Disponível: http://dx.doi.org/10.1590/S198314472013000300014

17. Barichello E, Sawada NO, Sonobe HM, Zago MMF. Qualidade do sono em pacientes submetidos à cirurgia oncológica. Rev. Latino-Am. Enfermagem. 2009;17(4):481-8.

18. Pozer MZ, Silva TA, Regino PA, Fernandes Junior PC, Silva SR. Sinais e sintomas de mielodepressão por quimioterapia no domicílio, entre portadoras de câncer ginecológico. Cienc. cuid. saude. 2012;11(2):336-42.

19. Ferreira NMLA, Souza MBB, Costa DB, Silva AC. Integridade cutâneo-mucosa: implicações para a família no cuidado domiciliário ao doente com câncer. Rev. enferm. UERJ. [Internet] 2009;17(2) [acesso em 04 abr 2014]. Disponível: http://www.facenf.uerj.br/ v17n2/v17n2a19.pdf

20. Santos DB, Vieira EM. Imagem corporal de mulheres com câncer de mama: uma revisão sistemática da literatura. Ciênc. saúde colet. 2011;16(5):2511-22.

21. Bordinhão RC. Processo de enfermagem em uma unidade de tratamento intensivo à luz da teoria das necessidades humanas básicas [dissertação]. Porto Alegre: Universidade Federal do Rio Grande do Sul; 2010.

22. Bittencourt AR, Alves DY, Luzia NS, Menezes MFB, Sória DAC. A temática da imagem corporal na produção científica nacional da enfermagem: um destaque para a pacientes com câncer. Rev. bras. cancerol. 2009;55(3):271-8.

23. Fernandes WC, Kimura M. Qualidade de vida relacionada à saúde de mulheres com câncer de colo uterino Rev. Latino-Am. Enfermagem. 2010;18(3):6572.

24. Guerrero GP, Zago MMF, Sawada NO, Pinto MH. Relação entre espiritualidade e câncer: perspectiva do paciente. Rev. bras. enferm. 2011;64(1):53-9. 